\title{
Helicobacter pylori Treatment Strategies in Singapore
}

\author{
Tiing Leong Ang and Daphne Ang \\ Department of Gastroenterology and Hepatology, Changi General Hospital, Singapore
}

\author{
Article Info \\ Received September 17, 2019 \\ Revised October 9, 2019 \\ Accepted October 22, 2019 \\ Published online December 30, 2019

\section{Corresponding Author} \\ Tiing Leong Ang \\ ORCID https://orcid.org/0000-0001-9993-8549 \\ E-mail ang.tiing.leong@singhealth.com.sg
}

\begin{abstract}
The management of Helicobacter pylori infection in Singapore remains a clinical challenge. Similar to other regions, there has been an increase in antibiotic resistance rates through the years. Nonetheless, over the past two decades, clarithromycin-based triple therapy has continued to be used as the first line treatment option, with an eradication rate exceeding $90 \%$, although the accepted treatment duration must now be lengthened from 1 to 2 weeks to maintain efficacy. Concomitant and sequential therapies did not demonstrate superiority over standard triple therapy. Current empiric second line treatment utilizes either bismuth-based quadruple therapy or levofloxacin-based triple therapy, but outcomes remain less than ideal. Identifying options to further improve treatment success rates is challenging. Strategies being considered include the use of potent acid suppressants, such as vonoprazan, and $H$. pylori culture and antibiotic susceptibility testing-guided therapy. (Gut Liver 2021;15:13-18)
\end{abstract}

Key Words: Helicobacter pylori; Eradication; Antibiotic resistance

\section{INTRODUCTION}

Singapore is a multiethnic Asian city-state, with a total population of 5.64 million in 2018, of whom 1.69 million are expatriates and migrant workers and 3.99 million are residents, accounting for 3.47 million Singapore citizens and 0.52 million permanent residents respectively. ${ }^{1}$ Based on the last population census conducted in 2010, amongst the resident population, the three major ethnic groups are Chinese (74.1\%), Malays (13.4\%) and Indians $(9.2 \%)^{2}{ }^{2} \mathrm{He}-$ licobacter pylori infection is common within the resident population. The high percentage of nonresident population poses an additional management challenge, as some of these individuals may come from regions with higher population prevalence rates of $H$. pylori infection, or with different antibiotic resistance patterns. The purpose of this review is to provide an updated report about the epidemiology of $H$. pylori infection in Singapore and its clinical burden, followed by an examination of the factors influencing treatment outcomes, and a review of the past, present and possible future strategies for $H$. pylori eradication in Singapore. These issues may mirror what is happening globally, but are contextualized to what is relevant for Singapore.

\section{EPIDEMIOLOGY OF H. PYLORI INFECTION} IN SINGAPORE

H. pylori infection is commonly encountered in clinical practice in Singapore. It is routinely screened for during upper endoscopy. For patients with dyspepsia who do not undergo upper endoscopy, carbon urea breath test, or less frequently $H$. pylori stool antigen, are performed. H. pylori serology is also frequently tested as part of health screening in the primary care setting for asymptomatic individuals.

In a brief report published in the local epidemiology news bulletin in 1996 (Epidemiol News Bull 1996;22:3132 ), the serology of 2,626 individuals aged between 6 months and 70 years were tested for $H$. pylori. The serology was obtained from healthy individuals who were blood donors and from hospitalized pediatric patients without gastrointestinal diseases. The serology was positive in $31.4 \%(\mathrm{n}=824)$. No gender difference was noted. The seroprevalence of $H$. pylori infection increased progressively with age from $3 \%$ in children below 5 years of age to $71 \%$ in adults above 65 years old. This study showed that the prevalence of $H$. pylori infection in Singapore had a pattern similar to that found in developed countries. ${ }^{3}$ A later study 
published in 2005 was based on a randomized community health survey of 11,000 Singaporean households conducted by the Ministry of Health, Singapore in 1998 that was representative of the local demographics. From this survey, a total of 7,000 asymptomatic healthy individuals were further randomly selected to participate in a health screening that involved answering a questionnaire and having blood tests. From this cohort, a similar number of subjects of both genders in two age groups: 25 to 39 years, and 55 to 69 years, from the Chinese, Malay and Indian respondents were then recruited, matching subjects for age, gender and race, yielding a total of 595 sera. This study reported that the H. pylori seroprevalence was similar between Chinese (46.3\%) and Indian (48.1\%) respondents, but significantly lower among Malay (27.9\%) respondents. This racial difference persisted when the analysis was stratified based on gender. In Malay men the seroprevalence was $30.1 \%$, while that in Malay women was $26 \%$. In contrast, amongst the Chinese respondents it was $46.8 \%$ and $45.8 \%$ for male and female subjects, respectively, while among the Indian respondents it was $51.1 \%$ and $45.1 \%$ for male and female subjects, respectively. ${ }^{4}$ More updated population-based epidemiological data are not available but $H$. pylori infection is still commonly encountered in clinical practice and remains a management problem.

\section{CLINICAL BURDEN OF H. PYLORI INFECTION}

As H. pylori infection results in chronic dyspepsia, peptic ulcer disease and gastric malignancies, eradication is universally recommended. ${ }^{5-11}$ Notwithstanding that many conditions are multifactorial in etiology, with more than one predisposing factor, such as the use of nonsteroidal anti-inflammatory drugs and antiplatelet therapies in the context of peptic ulcer disease, or gastric cancer, where age, genetic predisposition and other environmental factors also play a role, $H$. pylori infection represents a single easily addressed risk factor that should be eliminated. Indeed, $H$. pylori eradication has been demonstrated to significantly reduce symptoms in patients with functional dyspepsia, ${ }^{12}$ and be effective for primary and secondary prevention of peptic ulcer disease ${ }^{13,14}$ and gastric adenocarcinoma. ${ }^{15}$

\section{FACTORS INFLUENCING TREATMENT SUCCESS}

It is crucial to understand the factors influencing treatment success in order to devise a successful treatment strategy. Established factors include adequate acid sup- pression, use of combination of antibiotics, antibiotic resistance, adequate treatment duration and treatment adherence.

In vitro, $H$. pylori grows best between $\mathrm{pH} 6.0$ and 8.0 in the absence of urea and between $\mathrm{pH} 4.0$ and 6.0 in the presence of urea in buffered media. In vivo the role of acid inhibition is to increase intragastric $\mathrm{pH}$ so as to promote $H$. pylori transition from stationary to log phase growth thus making it susceptible to antibiotics. ${ }^{16}$ Data have shown that proton pump inhibitors (PPI) are superior to histamine2-receptor antagonists, ${ }^{17}$ and that twice daily PPI is superior to once daily PPI, ${ }^{18}$ in $\mathrm{H}$. pylori eradication regimens.

Antibiotic resistance is now a major concern and reason for failure of empiric therapies. In particular, the effectiveness of clarithromycin based triple therapy for $H$. pylori eradication has decreased in many geographic regions, largely due to development of resistance to clarithromycin. ${ }^{19}$ A meta-analysis reported that clarithromycin resistance reduced the efficacy of triple therapy by an estimated $35 \%$ (95\% confidence interval [CI], $26.4 \%$ to $46.4 \%$ ), whereas in the context of nitroimidazoles, efficacy was reduced by $30 \%$ when part of amoxycillin and acid suppressant based triple therapy, by $26 \%$ in triple therapies containing a nitroimidazole, tetracycline and bismuth, and by only $14 \%$ when part of quadruple therapy containing bismuth, gastric acid inhibitor, and tetracycline. ${ }^{20}$

A 14-day treatment regimen is recommended by international guidelines. ${ }^{8-11}$ A meta-analysis reported that regardless of type and dose of antibiotics, increased duration of PPI triple therapy from 7 to 14 days significantly increased the $H$. pylori eradication rate $(72.9 \%$ vs $81.9 \%)$. The relative risk for $H$. pylori persistence was 0.66 (95\% CI, 0.60 to 0.74$)$ and the number needed to treat was $11(95 \%$ CI, 9 to 14$).{ }^{21}$ Patient adherence to prescribed therapy is another crucial factor and compliance rate of less than $80 \%$ reduces treatment efficacy. ${ }^{22}$

\section{THE EVOLUTION OF TREATMENT OVER THE PAST TWO DECADES}

The choice of empiric therapy in Singapore, to some extent, mirrors the global trend although variations exist. These factors include $H$. pylori antibiotic resistance patterns, types of medications available and accessibility to antibiotic susceptibility testing in clinical practice.

\section{First line treatment}

The initial recommended empirical first line treatment from the late 1990s to 2015 was 1-week clarithromycin based triple therapy, with metronidazole replacing amoxi- 
cillin in patients with penicillin allergy and replacing clarithromycin in patients with clarithromycin allergy. In a multicenter randomized study $(\mathrm{n}=246)$ published in 2000 which compared 1-week omeprazole, amoxicillin, clarithromycin (OAC) with omeprazole, amoxicillin, metronidazole $(\mathrm{OAM})$ and omeprazole, metronidazole, clarithromycin $(\mathrm{OMC})$, the eradication rates $( \pm 95 \% \mathrm{CI})$ were intention-to-treat (ITT)/per protocol (PP) analyses: OAC $87 \%$ ( $79 \%$ to $94 \%) / 94 \%$ ( $89 \%$ to $100 \%$ ); OAM $80 \%$ (70\% to $89 \%) / 91 \%$ ( $83 \%$ to $98 \%$ ); OMC $85 \%$ ( $77 \%$ to $93 \%) / 94 \%$ ( $88 \%$ to $100 \%)$. The difference in eradication rates between the three groups was not statistically significant $(\mathrm{p}=0.419){ }^{23}$ Published data based on earlier studies suggested similar efficacy for 1-week versus 2-week treatment duration. ${ }^{24}$

With the recognition that $H$. pylori antibiotic resistance was becoming a major global challenge, despite the initial high success of 1-week based triple therapy regimens, ${ }^{25}$ further objective data was required. Previously published studies had suggested the superiority of sequential and concomitant therapy over triple therapy. Sequential therapy consisted of 5 days of treatment with a PPI and amoxicillin followed by 5-day treatment with the PPI and clarithromycin and metronidazole. A meta-analysis based on mainly European studies showed that the success rate of sequential therapy compared with triple therapy was $92.8 \%$ to $96 \%$ versus $76.2 \%$ to $78.8 \%{ }^{26}$ Concomitant therapy comprised 10 days of PPI, amoxicillin, clarithromycin, and metronidazole. Another meta-analysis found concomitant therapy to be superior to triple therapy. ${ }^{27}$ In a randomized controlled study published in 2015 of 462 patients who were randomized to receive either 10-day clarithromycin based triple therapy or 10-day sequential or 10-day concomitant therapy, the eradication rates obtained for sequential therapy versus triple therapy versus concomitant therapy were: ITT analysis, $84.4 \%$ versus $83.2 \%$ versus $81.7 \%$ ( $\mathrm{p}=$ not significant [NS]); modified ITT analysis, $90.3 \%$ versus $92.1 \%$ versus $94.7 \%(\mathrm{p}=\mathrm{NS}$ ); PP analysis, $94.1 \%$ versus $92.8 \%$ versus $95.4 \%(\mathrm{p}=\mathrm{NS})$. Dual resistance and lack of compliance were predictors of treatment failure. ${ }^{28}$

In a time trend analysis of antibiotic resistance patterns over the last 15 years in Singapore, we observed a significant increase in resistance rates for metronidazole (2000 to $2002,24.8 \%$; 2012 to $2014,48.2 \%$; $\mathrm{p}<0.001$ ), clarithromycin (2000 to $2002,7.9 \%$; 2012 to $2014,17.1 \%$; $\mathrm{p}=0.022$ ), and levofloxacin (2000 to 2002, 5\%; 2012 to $2014,14.7 \%$; $\mathrm{p}=0.007)$. The resistance rates for tetracycline (2000 to $2002,5 \% ; 2012$ to $2014,7.6 \%$ ) and amoxicillin (2000 to 2002, 3\%; 2012 to 2014: 4.4\%) remained stable. ${ }^{29}$ In addition, there was also an increase in dual (2000 to 2002, 6.9\%; 2012 to $2014,9.4 \% ; \mathrm{p}=0.479)$ and triple antibiotic resistance rates (2000 to 2002, 0; 2012 to $2014,7.6 \%$; $\mathrm{p}<0.001$ ).
Overall, the most common dual and triple resistance patterns were metronidazole/clarithromycin (4.4\%) and metronidazole/clarithromycin/levofloxacin $(1.8 \%)$, respectively. ${ }^{29}$

International consensus guidelines now recommend that 2-week empiric first line therapy be either guided by antibiotic susceptibility testing, if available, or be based on local susceptibility data. Bismuth based quadruple therapy and concomitant therapy, which is a non-bismuth based quadruple therapy, are recommended. Clarithromycin based triple therapy is not recommended when local resistance rates exceed $15 \%$ unless local data demonstrated efficacy. ${ }^{8-11}$

A clinical audit carried out at our hospital (unpublished data) during the period 2017 to 2018 examined the efficacy of clarithromycin based triple therapy in 862 patients. Treatment success rates were $76.9 \%$ for 7 -day treatment $(\mathrm{n}=13), 88.3 \%$ for 10 -day treatment $(\mathrm{n}=300)$ and $92 \%$ for 14-day treatment $(\mathrm{n}=549)$. In the same cohort, 16 patients were prescribed 14-day bismuth based quadruple therapy and an eradication rate of $87.5 \%$ was achieved. When $14-$ day PPI, metronidazole and clarithromycin was prescribed due to penicillin allergy $(n=23)$ the treatment success rate was only $69.6 \%$. The efficacy of $H$. pylori eradication therapies depends on multiple factors, with resistance to antibiotics and poor patient compliance being the most relevant ones. These data were based on a retrospective review of treatment outcomes, and were not derived from a prospective randomized study with adequate sample size. As such, they provide a snapshot of actual real-world efficacy but cannot establish either equivalence or non-inferiority of 14-day triple therapy and bismuth based quadruple therapy.

The data from our randomized study comparing triple therapy and non-bismuth quadruple therapies, ${ }^{28}$ and the results from the clinical audit of treatment outcomes for 2017 to 2018, would suggest that currently in Singapore, 2-week clarithromycin based triple therapy remains a viable empiric first line treatment option. Both bismuth and non-bismuth quadruple therapies do not demonstrate any superiority over triple therapy. What is clear currently, especially in context of high metronidazole resistance rates, is that in patients with penicillin allergy, metronidazole should no longer replace amoxicillin in a clarithromycin based regimen, and that bismuth based quadruple therapy might be preferred.

\section{Second line treatment}

Currently used empiric second line therapies in Singapore are either 2-week bismuth based quadruple therapy or levofloxacin based triple therapy, which comprise PPI, 
levofloxacin and amoxicillin. ${ }^{30}$ An earlier small retrospective study $(n=53)$ from Singapore reported eradication rate of $82.2 \%$ when using 7 -day bismuth based quadruple therapy. ${ }^{31}$ In the same clinical audit that was performed for the period 2017 and 2018 at our center (unpublished data), treatment success with 2-week bismuth quadruple therapy $(\mathrm{n}=51)$ was $82.4 \%$, whereas success rate with 2 -week PPI, amoxicillin and levofloxacin $(n=11)$ was $90.9 \%$. There is certainly room for improvement and for research to determine whether success rates can be further optimized, such as preferential use of levofloxacin-based triple therapy, or the use of antibiotic susceptibility testing to guide treatment regimen.

\section{CHALLENGES AND FUTURE STRATEGIES}

H. pylori eradication outcomes has been graded as follows: $\mathrm{F}$ or unacceptable ( $\leq 80 \%)$, D or poor ( $81 \%$ to $84 \%$ ), C or fair ( $85 \%$ to $89 \%)$, B or good ( $90 \%$ to $95 \%$ ), and A or excellent (95\% to $100 \%)$. The ideal is to use therapies that score "excellent" (grade=A). Regimens scoring as B or "good" can be used if "excellent" results are not obtainable. ${ }^{32}$ In Singapore, with the change from 1- to 2-week treatment duration, clarithromycin based triple therapy appears to remain effective in the context of empiric first line therapy, notwithstanding the gradual rise in resistance rate of clarithromycin over time. However, based on the report card (B) grading, more work is needed to explore other options to further improve treatment success rates. The success rate of current empiric second line therapy is also less than ideal currently.

Currently routine pretreatment culture and antibiotic susceptibility testing is not part of the initial management of $H$. pylori infection. $H$. pylori infection is not an emergency that requires urgent treatment and thus it would seem logical to adopt culture-guided therapy. Conventionally this involved culture and antibiotic susceptibility testing of $H$. pylori isolates obtained from gastric biopsies. Although a diagnosis of $H$. pylori infection may not be immediately evident during endoscopy, there may be clues, such as the pattern of gastritis or presence of peptic ulcer disease, which should then prompt additional biopsies to be taken during endoscopy, which can then be sent for $H$. pylori culture and antibiotic susceptibility testing should the rapid urease test becomes positive. Lately, a polymerase chain reaction-based approach, which assesses point mutations responsible for antibiotic resistance from gastric biopsy samples, has emerged as an alternative option. However, this is only applicable to antibiotic resistance to clarithromycin, fluoroquinolone and tetracycline which occurs as a result of specific mutations in a small region of the responsible gene. ${ }^{33} \mathrm{~A}$ meta-analysis demonstrated that susceptibility-guided therapy was superior to empiric therapy. ${ }^{34}$ In the past, susceptibility testing of $H$. pylori infection was limited by availability. At present, it has become more affordable and is readily available in hospitals in Singapore. If the test is only performed after failure of empiric treatment, the patient would require repeat endoscopy and incur additional costs and procedure-related risks. Studies have shown that culture-directed treatment can be costeffective. ${ }^{35,36}$ There is a need to balance the cost of additional testing with the failure rate of empirical therapy and this approach will be more important when resistance rates increase further.

Studies have shown that bismuth-containing regimens were superior to non-bismuth regimens in regions with high clarithromycin resistance. ${ }^{37}$ Apart from a primary therapeutic effect, bismuth has synergistic activity with antibiotics to overcome antibiotic resistance. Addition of bismuth to clarithromycin based triple therapy has been shown to increase the eradication rate. ${ }^{38}$ One can consider addition of bismuth to triple therapy but more data are needed to establish its incremental value in Singapore.

Vonoprazan was approved by Health Sciences Authority, Singapore, in July 2018 for treatment of $H$. pylori infection. Vonoprazan can achieve greater acid suppression than current PPI. Studies conducted in Japan have demonstrated superior eradication rate compared to PPI based triple therapy. ${ }^{39,40}$ However, the dose of antibiotics (amoxicillin $750 \mathrm{mg}$, clarithromycin $200 \mathrm{mg}$ or $400 \mathrm{mg}$ twice daily) and treatment duration (1 instead of 2 weeks) used in Japan are different from other guidelines. A recent study reported that vonoprazan-based triple therapy was non-inferior to susceptibility-guided PPI-based triple therapy. ${ }^{41}$ The incremental value of vonoprazan remains to be objectively validated in the Singapore setting. To answer this question, a randomized controlled trial comparing 2-week PPI and 1-week vonoprazan-based triple therapy is currently underway (NCT03908619).

\section{CONCLUSION}

H. pylori infection remains a common clinical problem. Current empiric treatment using 2-week clarithromycin based triple therapy remains effective but the treatment success rate can be further optimized. Empiric second line options are 2-week bismuth based quadruple therapy or levofloxacin based triple therapy, but success remains suboptimal. Potential options being explored include the use of more potent acid suppressants such as vonoprazan, 
addition of bismuth to triple therapy, and culture-directed treatment strategies.

\section{CONFLICTS OF INTEREST}

No potential conflict of interest relevant to this article was reported.

\section{ORCID}

Tiing Leong Ang https://orcid.org/0000-0001-9993-8549 Daphne Ang https://orcid.org/0000-0001-9438-9587

\section{REFERENCES}

1. Singapore Department of Statistics. Population Trends 2018 [Internet]. Department of Statistics, Ministry of Trade \& Industry, Republic of Singapore; c2018 [cited 2019 Sep 8]. Available from: https://www.singstat.gov.sg/-/media/files/ publications/population/population2018.pdf.

2. Singapore Department of Statistics. Census of population 2010 [Internet]. Department of Statistics, Ministry of Trade \& Industry, Republic of Singapore; c2011 [cited 2019 Sep 8]. Available from: https://www.singstat.gov.sg/-/media/files/ publications/cop2010/census_2010_release3/cop2010sr3. pdf.

3. Fock KM. Helicobacter pylori infection: current status in Singapore. Ann Acad Med Singapore 1997;26:637-641.

4. Ang TL, Fock KM, Dhamodaran S, Teo EK, Tan J. Racial differences in Helicobacter pylori, serum pepsinogen and gastric cancer incidence in an urban Asian population. J Gastroenterol Hepatol 2005;20:1603-1609.

5. Fock KM, Katelaris P, Sugano K, et al. Second Asia-Pacific consensus guidelines for Helicobacter pylori infection. J Gastroenterol Hepatol 2009;24:1587-1600.

6. Kim SG, Jung HK, Lee HL, et al. Guidelines for the diagnosis and treatment of Helicobacter pylori infection in Korea, 2013 revised edition. J Gastroenterol Hepatol 2014;29:13711386.

7. Sugano K, Tack J, Kuipers EJ, et al. Kyoto global consensus report on Helicobacter pylori gastritis. Gut 2015;64:13531367.

8. Malfertheiner P, Megraud F, O’Morain CA, et al. Management of Helicobacter pylori infection-the Maastricht V/Florence Consensus Report. Gut 2017;66:6-30.

9. Mahachai V, Vilaichone RK, Pittayanon R, et al. Helicobacter pylori management in ASEAN: the Bangkok consensus report. J Gastroenterol Hepatol 2018;33:37-56.
10. Fallone CA, Chiba N, van Zanten SV, et al. The Toronto Consensus for the treatment of Helicobacter pylori infection in adults. Gastroenterology 2016;151:51-69.

11. Fallone CA, Moss SF, Malfertheiner P. Reconciliation of recent Helicobacter pylori treatment guidelines in a time of increasing resistance to antibiotics. Gastroenterology 2019;157: 44-53.

12. Zhao B, Zhao J, Cheng WF, et al. Efficacy of Helicobacter pylori eradication therapy on functional dyspepsia: a metaanalysis of randomized controlled studies with 12-month follow-up. J Clin Gastroenterol 2014;48:241-247.

13. Vergara M, Catalán M, Gisbert JP, Calvet X. Meta-analysis: role of Helicobacter pylori eradication in the prevention of peptic ulcer in NSAID users. Aliment Pharmacol Ther 2005; 21:1411-1418.

14. Gisbert JP, Khorrami S, Carballo F, Calvet X, Gené E, Dominguez-Muñoz JE. H. pylori eradication therapy vs. antisecretory non-eradication therapy (with or without longterm maintenance antisecretory therapy) for the prevention of recurrent bleeding from peptic ulcer. Cochrane Database Syst Rev 2004;(2):CD004062.

15. Lee YC, Chiang TH, Chou CK, et al. Association between Helicobacter pylori eradication and gastric cancer incidence: a systematic review and meta-analysis. Gastroenterology 2016;150:1113-1124.

16. Sachs G, Scott DR, Wen Y. Gastric infection by Helicobacter pylori. Curr Gastroenterol Rep 2011;13:540-546.

17. Gisbert JP, Khorrami S, Calvet X, Gabriel R, Carballo F, Pajares JM. Meta-analysis: proton pump inhibitors vs. H2-receptor antagonists: their efficacy with antibiotics in Helicobacter pylori eradication. Aliment Pharmacol Ther 2003; 18:757-766.

18. Vallve M, Vergara M, Gisbert JP, Calvet X. Single vs. double dose of a proton pump inhibitor in triple therapy for Helicobacter pylori eradication: a meta-analysis. Aliment Pharmacol Ther 2002;16:1149-1156.

19. Graham DY, Fischbach L. Helicobacter pylori treatment in the era of increasing antibiotic resistance. Gut 2010;59:11431153.

20. Fischbach L, Evans EL. Meta-analysis: the effect of antibiotic resistance status on the efficacy of triple and quadruple firstline therapies for Helicobacter pylori. Aliment Pharmacol Ther 2007;26:343-357.

21. Yuan Y, Ford AC, Khan KJ, et al. Optimum duration of regimens for Helicobacter pylori eradication. Cochrane Database Syst Rev 2013;(12):CD008337.

22. Molina-Infante J, Romano M, Fernandez-Bermejo M, et al. Optimized nonbismuth quadruple therapies cure most patients with Helicobacter pylori infection in populations with high rates of antibiotic resistance. Gastroenterology 2013;145:121-128 
23. Fock KM, Chelvam P, Lim SG. Triple therapy in the eradication of Helicobacter pylori in patients with duodenal ulcer disease: results of a multicentre study in South-East Asia. South-East Asia Multicenter Study Group. Aliment Pharmacol Ther 2000;14:225-231.

24. Wong BC, Xiao SD, Hu FL, et al. Comparison of lansoprazole-based triple and dual therapy for treatment of Helicobacter pylori-related duodenal ulcer: an Asian multicentre double-blind randomized placebo controlled study. Aliment Pharmacol Ther 2000;14:217-224.

25. Ang TL, Wang L, Ang D, Chiam P, Fock KM, Teo EK. Is there still a role for empiric first-line triple therapy using proton pump inhibitor, amoxicillin and clarithromycin for Helicobacter pylori infection in Singapore? Results of a time trend analysis. J Dig Dis 2013;14:100-104.

26. Gatta L, Vakil N, Leandro G, Di Mario F, Vaira D. Sequential therapy or triple therapy for Helicobacter pylori infection: systematic review and meta-analysis of randomized controlled trials in adults and children. Am J Gastroenterol 2009;104:3069-3079.

27. Essa AS, Kramer JR, Graham DY, Treiber G. Meta-analysis: four-drug, three-antibiotic, non-bismuth-containing "concomitant therapy" versus triple therapy for Helicobacter pylori eradication. Helicobacter 2009;14:109-118.

28. Ang TL, Fock KM, Song M, et al. Ten-day triple therapy versus sequential therapy versus concomitant therapy as firstline treatment for Helicobacter pylori infection. J Gastroenterol Hepatol 2015;30:1134-1139.

29. Ang TL, Fock KM, Ang D, Kwek AB, Teo EK, Dhamodaran S. The changing profile of Helicobacter pylori antibiotic resistance in Singapore: a 15-year study. Helicobacter 2016;21:261-265.

30. Chew CA, Lye TF, Ang D, Ang TL. The diagnosis and management of H. pylori infection in Singapore. Singapore Med J 2017;58:234-240.

31. Ang TL, Fock KM, Ng TM, Teo EK, Chua TS, Tan YL. Efficacy of quadruple therapy for Helicobacter pylori eradication after failure of proton pump inhibitor based triple therapy in Singapore. Chin J Dig Dis 2003;4:35-39.
32. Graham DY, Lu H, Yamaoka Y. A report card to grade Helicobacter pylori therapy. Helicobacter 2007;12:275-278.

33. Nishizawa T, Suzuki H. Mechanisms of Helicobacter pylori antibiotic resistance and molecular testing. Front Mol Biosci 2014;1:19.

34. Vécsei A, Innerhofer A, Binder C, et al. Stool polymerase chain reaction for Helicobacter pylori detection and clarithromycin susceptibility testing in children. Clin Gastroenterol Hepatol 2010;8:309-312.

35. Breuer T, Graham DY. Costs of diagnosis and treatment of Helicobacter pylori infection: when does choosing the treatment regimen based on susceptibility testing become cost effective? Am J Gastroenterol 1999;94:725-729.

36. Kwon YH, Kim N, Lee JY, et al. Comparison of the efficacy of culture-based tailored therapy for Helicobacter pylori eradication with that of the traditional second-line rescue therapy in Korean patients: a prospective single tertiary center study. Scand J Gastroenterol 2016;51:270-276.

37. Dore MP, Lu H, Graham DY. Role of bismuth in improving Helicobacter pylori eradication with triple therapy. Gut 2016; 65:870-878.

38. Ko SW, Kim YJ, Chung WC, Lee SJ. Bismuth supplements as the first-line regimen for Helicobacter pylori eradication therapy: systemic review and meta-analysis. Helicobacter 2019;24:e12565.

39. Murakami K, Sakurai Y, Shiino M, Funao N, Nishimura A, Asaka M. Vonoprazan, a novel potassium-competitive acid blocker, as a component of first-line and second-line triple therapy for Helicobacter pylori eradication: a phase III, randomised, double-blind study. Gut 2016;65:1439-1446.

40. Li M, Oshima T, Horikawa T, et al. Systematic review with meta-analysis: vonoprazan, a potent acid blocker, is superior to proton-pump inhibitors for eradication of clarithromycinresistant strains of Helicobacter pylori. Helicobacter 2018;23: e12495.

41. Tanabe H, Yoshino K, Ando K, et al. Vonoprazan-based triple therapy is non-inferior to susceptibility-guided proton pump inhibitor-based triple therapy for Helicobacter pylori eradication. Ann Clin Microbiol Antimicrob 2018;17:29. 